\title{
A Nomogram to Predict Intra-Spinal Canal Cement Leakage Among Elderly Patients with Spine Metastases: An Internal-Validated Model
}

\author{
Xuedong Shi ${ }^{1} *$ \\ Yunpeng Cui ${ }^{1} *$ \\ Yuanxing Pan' \\ Bing Wang' \\ Mingxing Lei ${ }^{2,3}$ \\ 'Department of Orthopedic Surgery, \\ Peking University First Hospital, Beijing, \\ I00032, People's Republic of China; \\ ${ }^{2}$ Department of Orthopedic Surgery, \\ Hainan Hospital of Chinese PLA General \\ Hospital, Sanya, 572013, People's \\ Republic of China; ${ }^{3}$ Chinese PLA Medical \\ School, Beijing, I00853, People's Republic \\ of China
}

*These authors contributed equally to this work
Correspondence: Xuedong Shi Department of Orthopedic Surgery, Peking University First Hospital, No. 8 Xishiku Street, Xicheng District, Beijing, 100032, People's Republic of China

Tel +86 10-835722II

Email 172998774@qq.com;

xuedongs@hotmail.com

Mingxing Lei

Department of Orthopedic Surgery,

Hainan Hospital of Chinese PLA General

Hospital, Jianglin Road, Haitang District,

Sanya, 572013, People's Republic of China

Tel +86-18811772189

Email 825979020@qq.com
Purpose: This study aimed to assess the risk variables for predicting intra-spinal canal cement leakage, especially among elderly patients with spine metastases after being treated with percutaneous vertebroplasty (PVP). Furthermore, we proposed and validated a nomogram to stratify risks of intra-spinal canal cement leakage.

Methods: We retrospectively analyzed 163 elderly patients (age $\geqq 65$ years) with spine metastases who underwent PVP. Patients were randomly divided into a training cohort $(n=100)$ and a validation cohort $(n=63)$. The multivariate logistic regression analysis was used to screen potential risk variables in the training cohort. Significant risk variables were included in the nomogram, and the nomogram was developed according to the estimates of the each included variable. The predictive effectiveness of the nomogram was validated using discrimination and calibration performance.

Results: The overall prevalence of intra-spinal canal cement leakage was $9.82 \%(16 / 163)$. In the training cohort, female patients $(14.71 \%, 5 / 34)$ showed a higher rate of intra-spinal canal cement leakage as compared with male patients $(4.55 \%, 3 / 66)$. The nomogram consisted of sex, cortical osteolytic destruction in posterior wall, and load-bearing lines of spine. The nomogram had acceptable discrimination, with the area under the receiver operating characteristic (AUROC) of 0.75 in the training cohort, 0.64 in the validation cohort, and 0.69 in the entire cohort, and also showed favorable calibration based on the goodness-of-fit test. According to the nomogram, three risk groups were developed: the low risk group had an actual probability of $7.03 \%$, the medium risk group was $11.54 \%$, and high risk group was 44.44\%. The difference between the three groups was significant $(\mathrm{P}<0.01)$.

Conclusion: Intra-spinal canal cement leakage after PVP is not scarce among elderly patients. We proposed and internally validated a nomogram that is capable of calculating the risk of intra-spinal canal cement leakage among elderly patients with spine metastases. Careful surgical plan should be conducted among patients with a high risk of developing intra-spinal canal cement leakage.

Keywords: elderly patients, spine metastases, percutaneous vertebroplasty, cement leakage, nomogram

\section{Introduction}

Spine metastasis is a common condition among elderly patients with advanced cancer and is characterized by the symptoms of severe back pain, incontinence, and even disability, which significantly affects patients' quality of life. Appropriate treatments for spine metastases need a multidisciplinary approach. A prospective study in 2005 showed that decompressive surgery combined with radiotherapy was 
superior to radiotherapy alone in terms of survival and functional outcome. ${ }^{1}$ Therefore, this therapeutic method was gradually regarded as a standard in the treatment of spine metastases. However, several studies have reported that the associated complications of this surgery are cause for significant concern. ${ }^{2-4}$ Further, patients require more recovery time, which could lead to delays in receiving subsequent systematic primary cancer treatments. Moreover, as elderly patients usually have other underlying comorbidities and relatively poor immune systems, they may need additional time to recover. Therefore, an increasing number of patients with spine metastases are inclined to receive percutaneous vertebroplasty (PVP) instead.

PVP, widely applied in osteoporotic, neoplastic and traumatic vertebral compression fractures, ${ }^{5}$ facilitates pain relief, immediate stability, and early mobilization, ${ }^{6}$ mainly due to being a minimally invasive procedure. The control of bone cement distribution during surgery can be beneficial to reduce the risks of recompression. ${ }^{7}$ However, cement leaks were the most common complication of PVP, and the incidence of cement leakage could be up to $77 \% .^{8-10} \mathrm{~A}$ meta-analysis regarding all clinical complications of PVP showed that $66 \%$ cases were associated with the cement leakage. ${ }^{11}$ Recent studies reported that the prevalence of intra-spinal canal cement leakage ranged from $2.70 \%$ to $13.58 \%$ among patients with osteoporotic vertebral fractures treated with PVP. ${ }^{12,13}$ Most cement leaks would not result in any clinical symptoms, but cement leakage into the intra-spinal canal could lead to spinal cord and/or nerve root compression that would subsequently induce radicular pain, neurological deficits, to name just a few. ${ }^{14,15}$ Under such circumstances, emergency surgery is warranted. Therefore, prediction of cement leaks would be greatly helpful to prevent and reduce the occurrence of cement leakage.

Notably, risk variables associated with cement leakage could be used for accurate prediction. Some risk variables were proved to be significantly associated with cement leakage, including the fracture severity grade, cement viscosity, injected cement volume, vertebral body wall incompetence, cortical disruption, and a history of pulmonary diseases. ${ }^{9,16,17}$ However, researches about risk variables particularly to predict intra-spinal canal cement leakage is limited. Moreover, analysis of elderly population-specific risk variables was still more inadequate, as the majority of published literature discussing risk variables for cement leakage was not specially designed for elderly patients.

Therefore, we aimed to assess the risk variables to predict intra-spinal canal cement leakage especially in elderly patients with spine metastases who received PVP. Furthermore, we developed and validated a risk model to stratify risks of intra-spinal canal cement leakage in elderly patients with spine metastases.

\section{Methods}

\section{Study Setting}

This study retrospectively analyzed 163 elderly patients with spine metastases who underwent PVP at the orthopedic department of our hospital between January 2010 and January 2019. The surgical indications were patients with severe and/or uncontrolled back pain and corresponding symptoms that were not significantly relieved after conservative treatments. If patients were intolerable to open surgery, they could also be treated with PVP owing to minimal invasion and fast recovery of this technique. If patients underwent more than one PVP at a different time, we only analyzed the first operation. All patients were treated by the same team of spine surgeons. Both paper and electronic medical records were reviewed, and the data were collected by two reviewers to guarantee data quality and consistency. This study was in accordance with the Declaration of Helsinki. The Ethics Committee Board of our Hospital approved this study and waived patient consent, because all data were anonymized and the study was retrospective in nature.

\section{Inclusion and Exclusion Criteria}

The following inclusion criteria were applied: (1) patients with an age of 65 or above years; (2) diagnosis of spine metastases which were confirmed by imaging; (3) patients treated with PVP; (4) mixed or osteolytic lesions in the involved vertebrae; (5) severe or uncontrolled back pain due to metastatic tumors.

The exclusion criteria were as follows: (1) open decompressive spine surgery; (2) intramedullary metastases; (3) history of PVP because of other causes such as primary tumor, trauma, osteoporosis, and/or angioma; (4) severe radiculopathy and corresponding declining Frankel grades; (5) infection in the involved vertebrae and/or back skin at the corresponding level. 


\section{Primary Outcome and Risk Variables}

Intra-spinal canal cement leakage was defined as patients with epidural cement leakage which was confirmed by postoperative computed tomography (CT) examination, or radiography and fluoroscopy images recorded at the end of the surgery or intraoperative period. If patients suffered from radicular pain, neurological deficits, and/or dyspnea due to intra-spinal canal cement leakage, the corresponding clinical symptoms were also recorded.

The study included the following eleven potential risk variables for predicting intra-spinal canal cement leakage: age ( $\geq 65$ and $<70$ years vs $\geq 70$ and $<80$ years vs $\geq 80$ years); sex (male vs female); primary cancer types (rapid vs moderate vs slow) ${ }^{18}$ preoperative treatments (topical treatments vs systematic treatments vs no treatment); number of treated vertebrae levels ( 1 vs 2 vs 3 vs $\geq 4$ ); vertebrae collapse (no collapse vs $<50 \%$ vs $>50 \%$ ); ${ }^{19}$ cortical osteolytic destruction in the posterior wall (yes vs no); vertebral endplate fracture (yes vs no); the Bilsky scale ( 0 vs 1 vs 2 vs 3 , a higher score represents a more serious spinal cord compression); ${ }^{20}$ appearance of spine metastases (mixed lesions vs osteolytic lesions); and loadbearing lines of the spine (normal vs abnormal). The patient's age was defined as the time interval between the patient's birth and date of surgery. Topical treatments included topical analgesics and radiotherapy, while systematic treatments included oral or intravenous analgesics, targeted drugs, and chemotherapy.

\section{Developing the Nomogram}

When a prediction nomogram was created, the division of patients into at least the two groups was necessary. ${ }^{21,22}$ Thus, patients in the present were randomly divided into the training cohort $(n=100)$ and the validation cohort $(n=63)$. Patients in the training cohort were used to develop the nomogram, which enrolled the significant risk variables according to the multiple regression analysis (stepwise selection). Then, the nomogram was developed: $\mathrm{P}(\mathrm{Y}=1)=e^{\text {intercept }+\alpha x 1+\beta \times 2+\gamma \times 3} /\left(1+e^{\text {intercept }+\alpha x 1+\beta x 2+\gamma \times 3}\right)$. In the nomogram, $\alpha, \beta$, and $\gamma$ were the estimates of the included significant risk variables and $\mathrm{P}(\mathrm{Y}=1)$ was the predicted probability of intra-spinal canal cement leakage. The estimates were obtained when the significant variables entered the multivariate logistic analysis again.

\section{Evaluating the Nomogram's Performance}

The performance of the nomogram was assessed using discrimination and calibration in the training cohort, validation cohort, and the whole cohorts. The discrimination ability of the nomogram was used to distinguish patients with and without intra-spinal canal cement leakage, which was evaluated based on the area under the receiver operating characteristic (AUROC) curve and discrimination slope. A C-value $>0.7$ represents a good nomogram in the AUROC curve. Discrimination slope was defined as the difference between the mean predicted probability in patients with and without intra-spinal canal cement leakage. The calibration ability of the nomogram was defined as its accuracy between the predicted probability of intra-spinal canal cement leakage and the actual observed probability. The calibration ability of the nomogram was evaluated based on the calibration curve and goodness-of-fit statistics. A P $>0.05$ in the goodness-of-fit statistics indicated good calibration.

\section{Risk Stratification}

Each patient had an actual probability of intra-spinal canal cement leakage $(0.00 \%$ vs $100.00 \%)$ and could obtain a predicted probability, which was calculated using the nomogram. Then, the whole cohorts were divided into the three risk groups: the low risk group, the medium group, and the high risk group, and we further compared the difference of actual probability and predicted probability of intra-spinal canal cement leakage between the three risk groups. Statistical significances indicated acceptable stratification.

\section{Statistical Analysis}

Categorical variables were presented with proportions. Univariate and multivariate analysis was used to analyze the potential risk variables. The difference between the three risk groups was evaluated using the chi-square test. All statistical analyses were performed in SAS 9.4 software (SAS Institute Inc., Cary, NC) for Windows XP. A P $\leq 0.05$ was considered to indicate statistical significances.

\section{Results Patient's Basic Characteristics}

The study included 163 elderly patients with a mean age of $74.77 \pm 6.72$ years. Men accounted for $60.74 \%$ of the entire cohort. Rapid growth cancers $(54.60 \%)$ were the most common primary cancer types, followed by slow growth cancers $(32.52 \%)$. The majority of patients received treatments before surgery: $35.58 \%$ patients were treated with topical treatments and $33.74 \%$ patients were treated with systematic treatments. Overall, 59.51\% patients underwent 
treatment at more than one vertebral level, 58.28\% patients had no vertebral collapse, $62.58 \%$ patients had no cortical osteolytic destruction in the posterior wall, $82.82 \%$ patients had no vertebral endplate fracture, $80.37 \%$ patients had a Bilsky scale of $0,74.85 \%$ patients had osteolytic lesions, and $84.66 \%$ patients had normal loadbearing lines of spine (Table 1). Intra-spinal canal cement leakage was observed in 9.82\% (16/163) patients (Case report is presented in Figure 1). No pulmonary or cardiac embolism was observed in the cohorts.

\section{Development of the Nomogram}

The multiple logistic regression analysis found that sex (odds ratio $[\mathrm{OR}]=9.43,95 \% \mathrm{CI}$ : $1.00-89.69, \mathrm{P}=0.05$ ), cortical osteolytic destruction in the posterior wall $(\mathrm{OR}=12.35$, 95\% CI: 1.35-113.36, $\mathrm{P}=0.03)$ and loadbearing lines of the spine $(\mathrm{OR}=20.91,95 \% \mathrm{CI}$ : $1.03-$ 423.34, $\mathrm{P}=0.05$ ) were significantly associated with intraspinal canal cement leakage (Table 2). Therefore, the three risk variables were included to develop the nomogram. The estimates were obtained by putting the three significant risk variables re-enter into the multivariate logistic regression analysis. A nomogram was created as follows (Table 3): $\quad \mathrm{P}(\mathrm{Y}=1) \quad=\mathrm{e}^{-5.53+1.27 \times 1+1.80 \times 2+1.43 \times 3} /(1$ $\left.+\mathrm{e}^{-5.53+1.27 \times 1+1.80 \times 2+1.43 \times 3}\right)$, where $\mathrm{x} 1-\mathrm{x} 3$ indicate sex, cortical osteolytic destruction in the posterior wall, and loadbearing lines of the spine, respectively. $\mathrm{P}(\mathrm{Y}=1)$ indicates the predicted probability of occurrence of intra-spinal canal cement leakage. The scores in the nomogram were assigned based on the original data. An example of how to use the nomogram is given in Table 3. A calculator was developed to promote clinical utility (Supplementary file).

\section{Performance of the Model}

The AUROC of the nomogram was 0.75 in the training cohort (Figure 2A and Table 4), 0.64 in the validation cohort (Figure 2B), and 0.69 in the whole population (Figure 2C). The corresponding discrimination slopes were 0.16 (95\% CI: 0.09-0.22, Figure 3A), 0.07 (95\% CI: $-0.01-0.15$, Figure 3B), and 0.12 (95\% CI: $0.07-$ 0.17 , Figure $3 \mathrm{C}$ ), respectively. The results indicated that the nomogram had acceptable and favorable discrimination ability.

The calibration curve presented good concordance between predicted and observed risk in the training cohort (Figure 4A), the validation cohort (Figure 4B), and the
Table I Patient's Demographic and Clinical Characteristics

\begin{tabular}{|c|c|}
\hline Characteristics & $\begin{array}{l}\text { Patients } \\
(n=163)\end{array}$ \\
\hline \multicolumn{2}{|l|}{ Age (years) } \\
\hline$\geq 65$ and $<70$ & $28.22 \%(46 / 163)$ \\
\hline$\geq 70$ and $<80$ & $47.24 \%(77 / / 63)$ \\
\hline$\geq 80$ & $24.54 \%(40 / 163)$ \\
\hline \multicolumn{2}{|l|}{ Sex } \\
\hline Male & $60.74 \%(99 / 163)$ \\
\hline Female & $39.26 \%(64 / 163)$ \\
\hline \multicolumn{2}{|l|}{ Primary cancer types } \\
\hline Slow growth & $32.52 \%(53 / 163)$ \\
\hline Moderate growth & $12.88 \%(21 / 163)$ \\
\hline Rapid growth & $54.60 \%(89 / 163)$ \\
\hline \multicolumn{2}{|l|}{ Preoperative treatments } \\
\hline Topical treatments & $35.58 \%(58 / 163)$ \\
\hline Systematic treatments & $33.74 \%(55 / 163)$ \\
\hline No treatment & $30.67 \%(50 / 163)$ \\
\hline \multicolumn{2}{|l|}{ Number of treated vertebrae levels } \\
\hline 1 & $40.49 \%(66 / 163)$ \\
\hline 2 & $22.09 \%(36 / 163)$ \\
\hline 3 & $16.56 \%(27 / 163)$ \\
\hline$\geq 4$ & $20.86 \%(34 / 163)$ \\
\hline \multicolumn{2}{|l|}{ Vertebrae collapse } \\
\hline No collapse & $58.28 \%(95 / 163)$ \\
\hline Less than $50 \%$ & $26.38 \%(43 / 163)$ \\
\hline More than $50 \%$ & $15.34 \%(25 / 163)$ \\
\hline \multicolumn{2}{|l|}{ Cortical osteolytic destruction in posterior wall } \\
\hline Yes & $37.42 \%(61 / 163)$ \\
\hline No & $62.58 \%(102 / 163)$ \\
\hline \multicolumn{2}{|l|}{ Vertebral endplate fracture } \\
\hline Yes & $17.18 \%(28 / 163)$ \\
\hline No & $82.82 \%(135 / 163)$ \\
\hline \multicolumn{2}{|l|}{ The Bilsky scale } \\
\hline 0 & $80.37 \%(131 / 163)$ \\
\hline 1 & $10.43 \%(17 / 163)$ \\
\hline 2 & $7.98 \%(13 / 163)$ \\
\hline 3 & $1.23 \%(2 / 163)$ \\
\hline \multicolumn{2}{|l|}{ Appearance of spine metastases } \\
\hline Mixed lesions & $25.15 \%(41 / 163)$ \\
\hline Osteolytic lesions & $74.85 \%(122 / 163)$ \\
\hline \multicolumn{2}{|l|}{ Load-bearing lines of spine } \\
\hline Normal & $84.66 \%(138 / 163)$ \\
\hline Abnormal & $15.34 \%(25 / 163)$ \\
\hline \multicolumn{2}{|l|}{ Intra-spinal canal cement leakage } \\
\hline Yes & $9.82 \%(16 / 163)$ \\
\hline No & $90.18 \%(147 / 163)$ \\
\hline
\end{tabular}



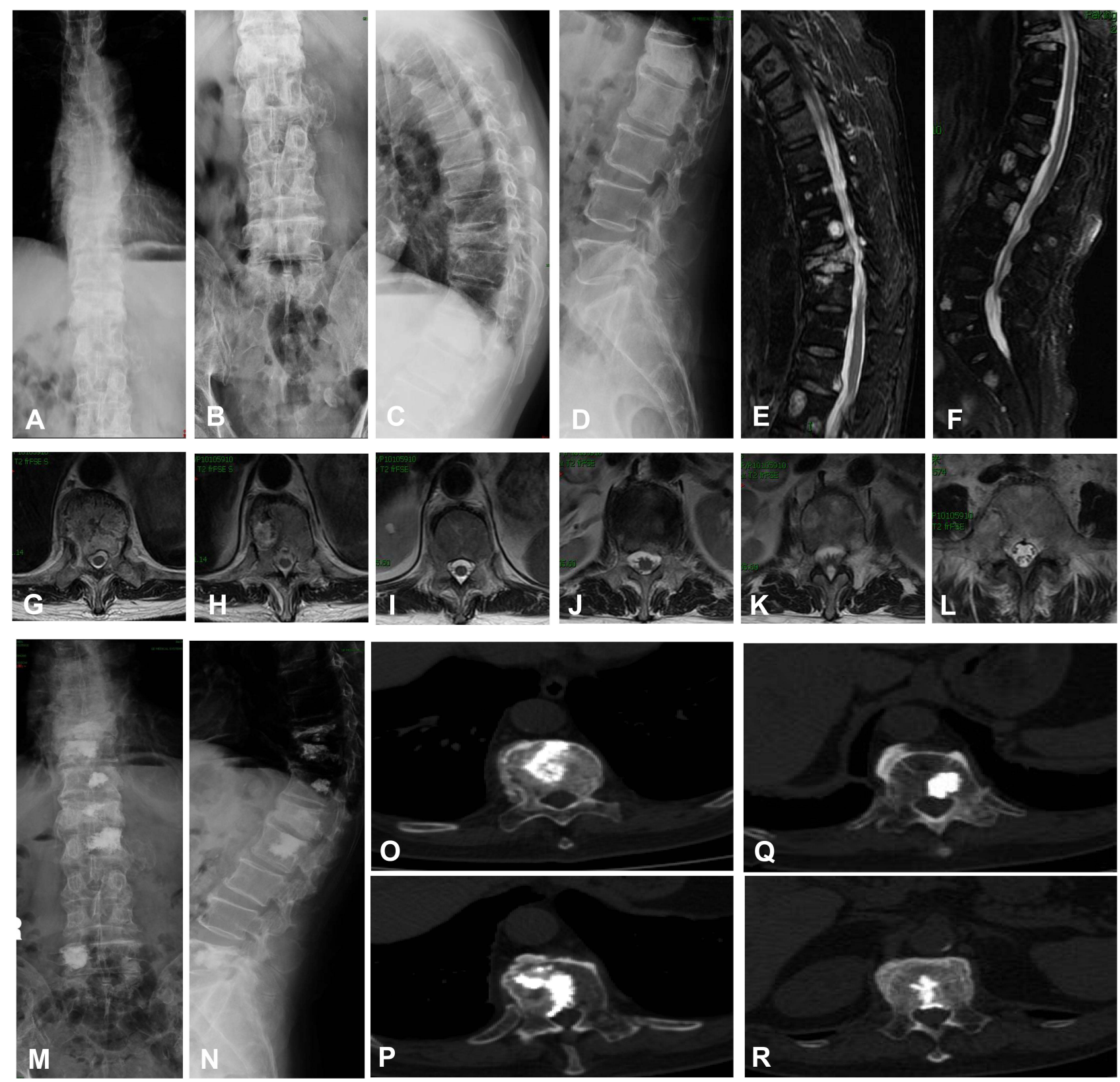

Figure I An 80-year-old man with spine metastases treated with PVP and suffered from intra-spinal canal cement leakage (before surgery). (A) Preoperative anteroposterior thoracic vertebral radiography showed vertebral collapse at TIO and LI; (B) Preoperative anteroposterior lumbar vertebral radiography revealed vertebral collapse at LI; (C) Preoperative lateral thoracic vertebral radiography showed vertebral collapse at TIO and LI; (D). Preoperative lateral lumbar vertebral radiography showed vertebral collapse at LI; (E) Preoperative sagittal thoracic vertebral MRI showed multiple spine metastases and vertebral collapse at TIO and LI; (F) Preoperative sagittal lumbar vertebral MRI showed multiple spine metastases and vertebral collapse at TIO and LI; (G) Preoperative transversal TIO MRI showed deformation of the dural sac, but no spinal cord or nerve root compression; (H) Preoperative transversal MRI at TII showed metastatic lesion; (I) Preoperative transversal MRI at TI2 showed metastatic lesion; (J) Preoperative transversal MRI at LI showed metastatic lesion; (K) Preoperative transversal MRI at L2 showed metastatic lesion; (L) Preoperative transversal MRI at L5 showed metastatic lesion; (M) Postoperative anteroposterior lumbar vertebral radiography revealed bone cement at TI0, TII, TI2, LI, L2, and L5; (N) Postoperative lateral vertebral radiography showed bone cement at TI0, TI I, TI2, LI, L2, and L5; (O) Postoperative transversal CT at TI0 showed distribution of bone cement; (P) Postoperative transversal CT at TII showed intra-spinal canal cement leakage; (Q) Postoperative transversal CT at TI2 showed intra-spinal canal cement leakage; (R) Postoperative transversal CT at LI showed intra-spinal canal cement leakage.

whole cohort (Figure 4C). The Hosmer-Lemeshow test also indicated that there was no significant statistical difference in the training cohort (Chi-square $=1.13, \mathrm{P}=0.77$ ), the validation cohort (Chi-square $=0.56, \mathrm{P}=0.97)$, and the whole patients (Chi-square $=2.12, \mathrm{P}=0.71$ ), suggesting the nomogram was relatively well calibrated. 
Table 2 Multivariate Logistic Regression Analyses of Potential Risk Variables for Predicting Intra-Spinal Canal Cement Leakage in Patients with Spine Metastases Treated with Percutaneous Vertebroplasty in the Training Group

\begin{tabular}{|c|c|c|c|c|c|}
\hline \multirow[t]{2}{*}{ Potential Risk Variables } & \multirow[t]{2}{*}{ Rates } & \multicolumn{2}{|c|}{$\begin{array}{l}\text { Univariate Logistic } \\
\text { Regression }\end{array}$} & \multicolumn{2}{|c|}{ Multivariate Logistic Regression } \\
\hline & & OR (95\% CI) & $\mathbf{P}$ & OR $(95 \% \mathrm{Cl})$ & $\mathbf{P}$ \\
\hline \multicolumn{6}{|l|}{ Age (years) } \\
\hline$\geq 65$ and $<70$ & $10.00 \%(3 / 30)$ & & & & \\
\hline$\geq 70$ and $<80$ & $9.09 \%(4 / 44)$ & $0.66(0.24-1.79)$ & 0.41 & $0.60(0.17-2.17)$ & 0.43 \\
\hline$\geq 80$ & $3.85 \%(1 / 26)$ & & & & \\
\hline \multicolumn{6}{|l|}{ Sex } \\
\hline Male & $4.55 \%(3 / 66)$ & $3.62(0.8 I-16.19)$ & 0.09 & $9.43(1.00-89.69)$ & 0.05 \\
\hline Female & $|4.7| \%(5 / 34)$ & & & & \\
\hline \multicolumn{6}{|l|}{ Primary cancer types } \\
\hline Slow growth & $8.82 \%(3 / 34)$ & & & & \\
\hline Moderate growth & $0.00 \%(0 / 15)$ & I.II $(0.50-2.5 \mathrm{I})$ & 0.79 & $1.45(0.29-7.16)$ & 0.65 \\
\hline Rapid growth & $9.80 \%(5 / 51)$ & & & & \\
\hline \multicolumn{6}{|l|}{ Preoperative treatments } \\
\hline Topical treatments & $5.56 \%(2 / 36)$ & & & & \\
\hline Systematic treatments & II.II\% (4/36) & $1.15(0.46-2.83)$ & 0.77 & $1.55(0.47-5.10)$ & 0.47 \\
\hline No treatment & $7.14 \%(2 / 28)$ & & & & \\
\hline \multicolumn{6}{|l|}{ Number of treated vertebrae levels } \\
\hline I & $10.26 \%(4 / 39)$ & & & & \\
\hline 2 & $12.50 \%(3 / 24)$ & $0.67(0.33-1.38)$ & 0.28 & $0.54(0.23-1.30)$ & 0.17 \\
\hline 3 & $0.00 \%(0 / 16)$ & & & & \\
\hline$\geq 4$ & $4.76 \%(1 / 21)$ & & & & \\
\hline \multicolumn{6}{|l|}{ Vertebrae collapse } \\
\hline No collapse & $10.00 \%(6 / 60)$ & & & & \\
\hline Less than $50 \%$ & $3.70 \%(1 / 27)$ & $0.69(0.22-2.18)$ & 0.53 & $0.28(0.03-3.23)$ & 0.31 \\
\hline More than $50 \%$ & $7.69 \%(1 / 13)$ & & & & \\
\hline \multicolumn{6}{|l|}{ Cortical osteolytic destruction in posterior wall } \\
\hline Yes & $15.79 \%(6 / 38)$ & $5.63(1.07-29.49)$ & 0.04 & $12.35(1.35-113.36)$ & 0.03 \\
\hline No & $3.23 \%(2 / 62)$ & & & & \\
\hline \multicolumn{6}{|l|}{ Vertebral endplate fracture } \\
\hline Yes & $5.88 \%(1 / 17)$ & $0.68(0.08-5.91)$ & 0.73 & I.7I (0.04-79.45) & 0.78 \\
\hline No & $8.43 \%(7 / 83)$ & & & & \\
\hline \multicolumn{6}{|l|}{ The Bilsky scale } \\
\hline 0 & $8.33 \%(7 / 84)$ & & & & \\
\hline I & $0.00 \%(0 / 1 \mathrm{I})$ & $1.10(0.33-3.72)$ & 0.87 & $0.74(0.11-4.99)$ & 0.76 \\
\hline 2 & $25.00 \%(1 / 4)$ & & & & \\
\hline 3 & $0.00 \%(0 / I)$ & & & & \\
\hline \multicolumn{6}{|l|}{ Appearance of spine metastases } \\
\hline Mixed lesions & $6.90 \%(2 / 29)$ & $1.25(0.24-6.57)$ & 0.80 & $0.12(0.00-4.55)$ & 0.25 \\
\hline Osteolytic lesions & $8.45 \%(6 / 71)$ & & & & \\
\hline \multicolumn{6}{|l|}{ Load-bearing lines of spine } \\
\hline Normal & $6.82 \%(6 / 88)$ & $2.73(0.49-15.42)$ & 0.25 & $20.91(1.03-423.34)$ & 0.05 \\
\hline Abnormal & $16.67 \%(2 / 12)$ & & & & \\
\hline
\end{tabular}

Abbreviations: $\mathrm{OR}$, odds ratio; $\mathrm{Cl}$, confidential interval. 
Table 3 The Nomogram to Calculate the Probability of IntraSpinal Canal Cement Leaks in Patients with Spine Metastases

\begin{tabular}{|l|c|c|}
\hline Included Variables & Scores & Estimates $^{\mathbf{a}}$ \\
\hline Intercept & & -5.53 \\
\hline Sex & & \\
$\quad$ Male & 2 & 1.27 \\
$\quad$ Female & & \\
\hline Cortical osteolytic destruction in posterior & & \\
wall & 1 & 1.80 \\
Yes & 0 & \\
No & & \\
\hline Load-bearing lines of spine & 0 & 1.43 \\
Normal & $\mathrm{I}$ & \\
Abnormal & \\
\hline
\end{tabular}

Notes: ${ }^{a}$ Indicates the estimates were obtained from the multivariate logistic analysis of the three significant variables. A formula was developed as follows: $\mathrm{P}(\mathrm{Y}=1)=$ $e^{-5.53+1.27 x 1+1.80 \times 2+1.43 \times 3} /\left(I+e^{-5.53+1.27 x 1+1.80 \times 2+1.43 \times 3}\right) ; x \mathrm{I}$ indicates gender; $x 2$ indicates cortical osteolytic destruction in posterior wall; $x 3$ indicates load-bearing lines of spine. $P(Y=I)$ indicates the predicted probability of occurrence of intra-spinal canal cement leakage. An example was shown as follows: If a female $(x \mid=2)$ patients with cortical osteolytic destruction in posterior wall $(x 2=1)$ and normal loadbearing lines of spine $(x 3=0)$ treated with percutaneous vertebroplasty, then the predicted probability of occurrence of intra-spinal canal cement leakage was $\mathrm{P}(\mathrm{Y}=\mathrm{I})$ $=e^{-5.53+1.27 * 2+1.80 * 1+1.43 * 0} /\left(\mathrm{I}+e^{-5.53+1.27 * 2+1.80 * 1+1.43 * 0}\right)=23.33 \%$.

\section{Risk Group Classification}

According to the predicted probability, which was obtained from the nomogram for each patient, we classified all the patients into the three risk groups (Table 5): Patients with a predicted probability of less than $10.00 \%$ were low risk groups and the actual probability was only $7.03 \%$, patients with a predicted probability of $10.00 \%$ to $24.99 \%$ were medium risk group and the actual probability was $11.54 \%$, and patients with a predicted probability of $25.00 \%$ or more were high risk group and the actual probability was as high as $44.44 \%$. The difference between the three risk groups was statistically different $(\mathrm{P}<0.01)$, which indicated a distinguished risk classification.

\section{Discussion}

Intra-spinal canal cement leakage was observed in $9.82 \%$ $(16 / 163)$ of elderly patients with spine metastases in the present study. Recent studies reported the prevalence of intra-spinal canal cement leakage ranged from $2.70 \%$ to $13.58 \%$ among patients with osteoporotic vertebral fractures treated with PVP. ${ }^{12,13}$ Intra-spinal canal cement leakage may lead to severe complications and even disability. Therefore, a risk model that can evaluate the risk of intraspinal canal cement leakage was clinically essential. We found that risk variables for predicting intra-spinal canal cement leakage included sex, cortical osteolytic destruction in the posterior wall, and load-bearing lines of the spine. The cortical osteolytic destruction in the posterior wall had already been considered as a significant predictor for intra-spinal canal cement leakage. ${ }^{23}$ Zhang et al ${ }^{12}$ have confirmed that preoperative Cobb angle affected the occurrence of intra-spinal canal cement leakage, which was consistent with our present study indicating that an abnormal load-bearing line of spine was associated with more cement leakage into spinal canal. We also found that female was associated with more intra-spinal canal cement leakage, which might be exampled by severe osteoporosis due to declined estrogen among elderly women.

Some risk variables associated with cement leakage were presented in other studies, including the fracture severity grade, cement viscosity, injected cement volume, integrity of vertebral walls, vertebral body wall incompetence, cortical disruption, vertebral cortical bone defect, bone density, and a history of pulmonary diseases. ${ }^{9,16,17,24-26}$ These risk variables for predicting cement leakage might be controversial, because (1) the definition of the above-mentioned variables varied in different studies, (2) the patients enrolled in these studies mostly had osteoporotic vertebral fracture instead of metastatic spinal disease alone, and (3) these studies investigated the potential risk variables for cement leakage, not specifically for intra-spinal canal cement leakage. Consequently, specific risk variables for predicting intraspinal canal cement leakage really needed further investigations.

The cortical osteolytic destruction in the posterior wall was regarded as an essential predictor for intra-spinal canal cement leakage. ${ }^{23} \mathrm{Zhu}$ et $\mathrm{al}^{27}$ found that patients with a cement volume of less than $3.5 \mathrm{~mL}$ and a unilateral approach were more likely to suffer from the spinal canal cement leakage. Previously, we also found that Bilsky scale was significantly associated with intraspinal canal cement leakage after analyzing 251 patients with spine metastases. ${ }^{28}$ In our present study, we found that sex, cortical osteolytic destruction in the posterior wall, and load-bearing lines of the spine were significant parameters. However, Chen et $\mathrm{al}^{25}$ found that sex was not significantly associated with cement leakage after analyzing 102 cases with osteoporotic vertebral fractures. This difference was likely because we specifically included elderly patients with spine metastases and especially investigated the relationship between potential clinical risk variables and intra-spinal canal cement leakage. 

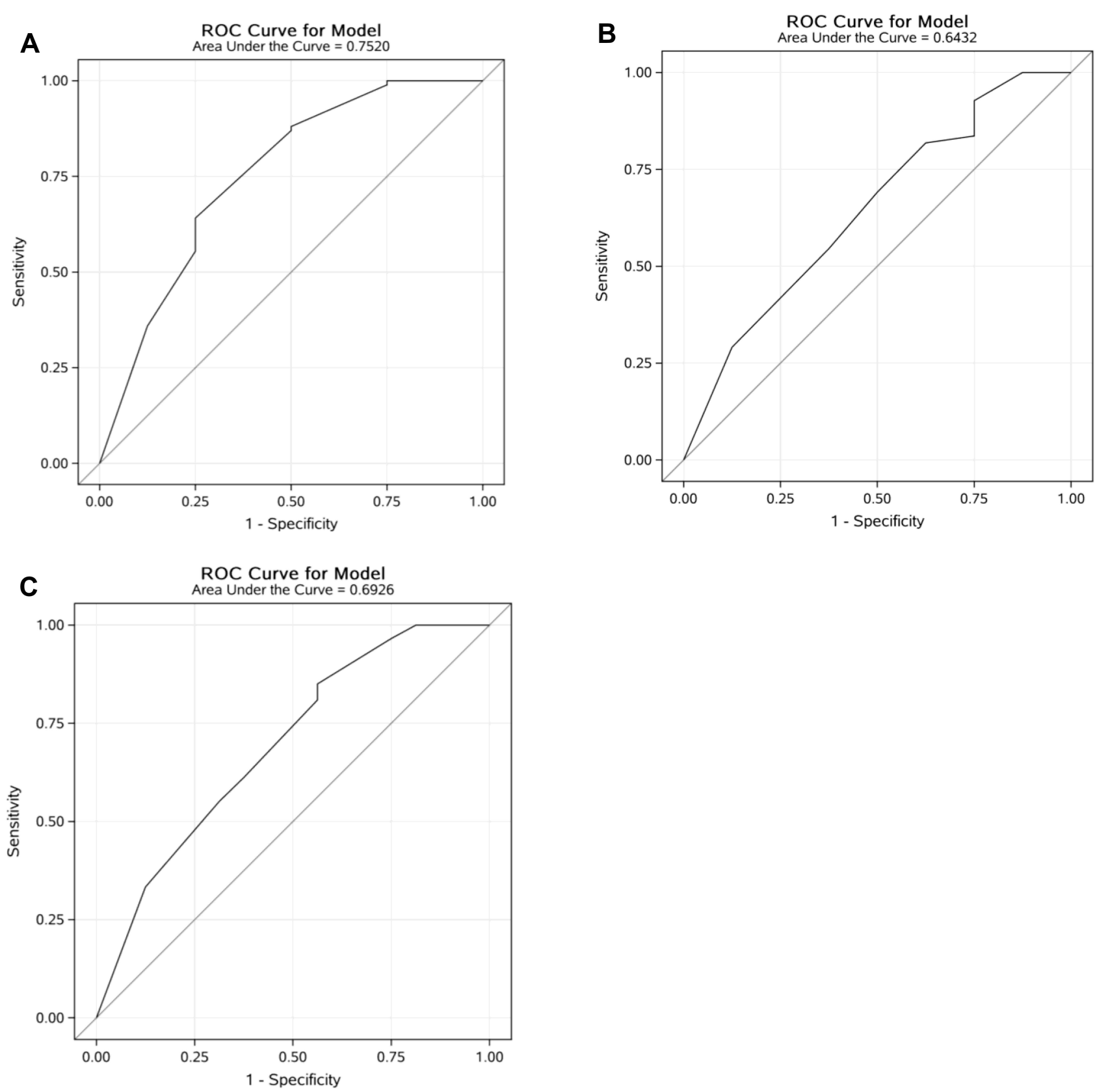

Figure 2 The area under the receiver operating characteristic (AUROC) curve for the nomogram: (A) the training group; (B) the validation group; (C) the both group.

Some studies have reported the pulmonary or cardiac Janssen et $\mathrm{al}^{29}$ reported that $1.82 \%(3 / 165)$ of patients cement embolism and the prevalence was relatively low: suffered from pulmonary cement embolism after treating

Table 4 Predictive Performance of the Nomogram

\begin{tabular}{|l|c|c|c|c|c|}
\hline \multirow{2}{*}{ Formula } & \multicolumn{3}{|c|}{ Discrimination Ability } & \multicolumn{2}{c|}{ Calibration Ability } \\
\cline { 2 - 6 } & AUROC & Slope & $\mathbf{9 5 \% ~ C l}$ & Chi-Square Value & P \\
\hline Training group & 0.75 & 0.16 & $0.09-0.22$ & 1.13 & 0.77 \\
Validation group & 0.64 & 0.07 & $-0.01-0.15$ & 0.56 & 0.97 \\
Both groups & 0.69 & 0.12 & $0.07-0.17$ & 2.12 & 0.71 \\
\hline
\end{tabular}

Notes: ${ }^{a}$ Indicates $\mathrm{P}$ value was obtained from the goodness-of-fit test.

Abbreviations: AUROC, area under the receiver operating characteristic curve; $\mathrm{Cl}$, confidential interval. 


\section{A}

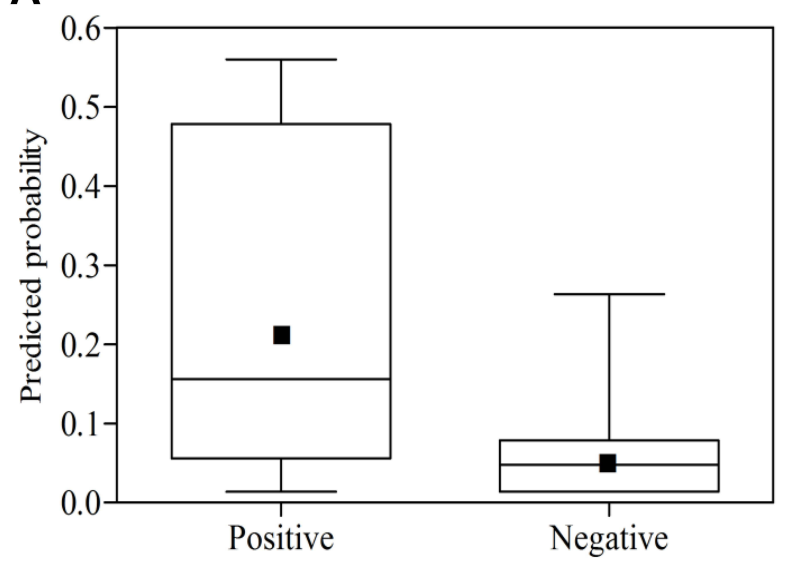

C

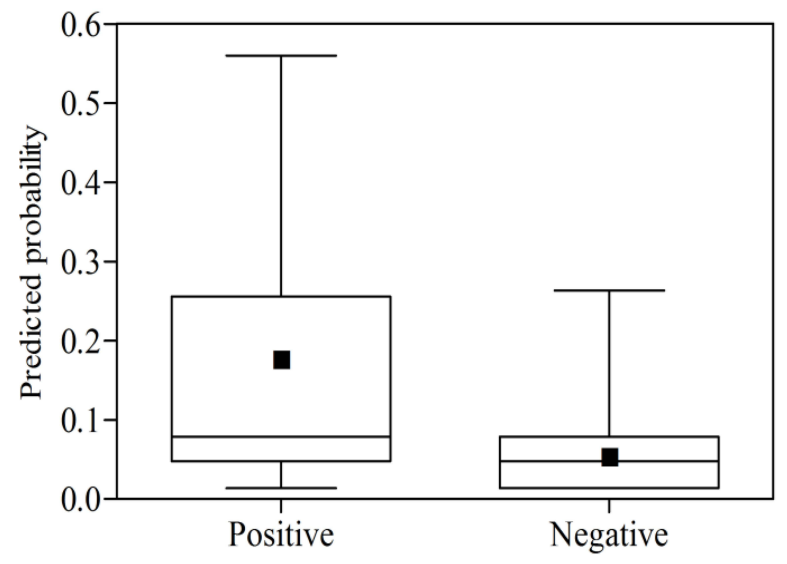

B

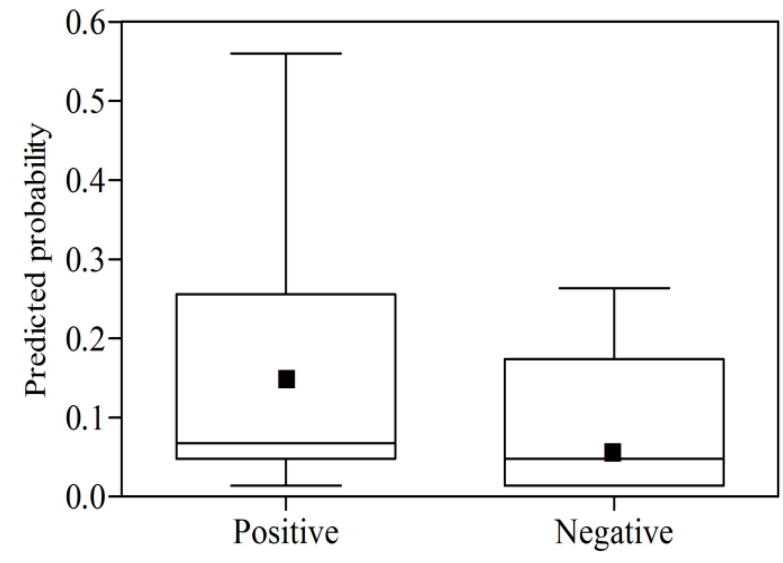

Figure 3 Box plots of predicted probabilities for the nomogram: (A) the training group; (B) the validation group; (C) the both group combined. Discrimination slope was the difference between the mean predicted probability with (positive events) and without (negative events) intra-spinal canal cement leakage. Black solid dots indicate the mean value of the predicted probability of intra-spinal canal cement leakage.

with cement augmented pedicle instrumentation of the thoracolumbar spine, and the embolism was verified on thoracic CT. More recently, Hsieh et $\mathrm{al}^{14}$ showed $0.24 \%$ (9/3812) of vertebroplasty procedures occurred pulmonary embolism. In our present study, no pulmonary or cardiac embolism was observed in our cohorts.

Furthermore, we proposed and validated a model based on the above three risk variables. The nomogram was concise and practical nomogram since it only consisted of three risk variables. This nomogram also showed acceptable discrimination and favorable calibration ability based on the AUROC and calibration curves. This model could effectively calculate the risk probability of intra-spinal canal cement leakage among elderly patients with spine metastases; thereby it might provide early information to patients about potential surgery complications and facilitate surgeons to design surgical strategies and plans. Stratification capacity of the model was also relatively excellent: the low risk groups had an actual probability of only $7.03 \%$, the medium risk group had an actual probability of $11.54 \%$, and the high risk group had an actual probability of up to $44.44 \%$. The difference between the three risk groups was statistically different $(\mathrm{P}<0.01)$, which indicated distinguished risk classification. Considering the high risk of intra-spinal canal cement leakage among the patients in the high risk group, we recommended that surgeons to pay more attention to carefully make surgical plans to avoid intra-spinal canal cement leakage, such as injecting appropriate volume of bone cement, increasing intraoperative fluoroscopy, and intraoperatively monitoring the flow of bone cement, while patients in the low risk groups had a relatively low risk of intra-spinal canal cement leakage, more emphases might need to be placed to care about other surgery-related complications, including vascular cement leakage and intervertebral disc cement leakage. The interventions for 

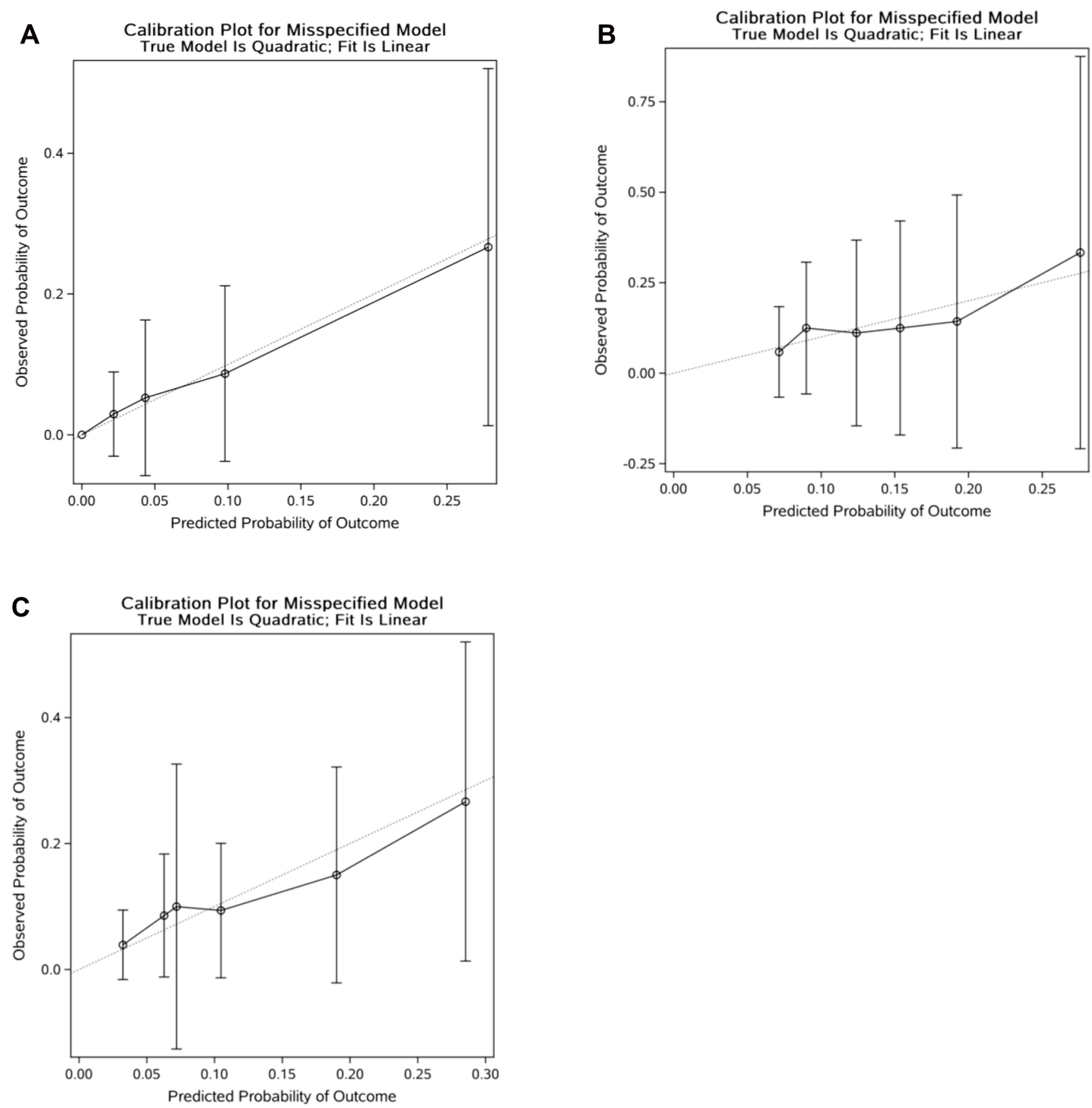

Figure 4 Plotting deciles of the predicted probability of intra-spinal canal cement leakage against the observed proportions for the nomogram: (A) the training group; (B) the validation group; $(\mathbf{C})$ the both groups combined. The $x$-axis is the predicted risk and the $y$-axis is the actual risk. The grey dotted lines indicate a perfect prediction by an ideal model. The solid black lines indicate the performance of the nomogram, and a closer fit to the diagonal dotted lines indicate a better prediction.

medium risk group should be balanced between the high risk group and the low risk group.

\section{Limitations}

The retrospective design of the study and the limited potential variables are some of the limitations of this study. The retrospective nature makes it difficult to avoid selection bias. Some potential variables such as the viscosity of bone cement were not investigated in the study. A higher bone cement viscosity was beneficial to prevent leakage. ${ }^{30}$ However, cement viscosity has no gold standard for assessment, because the storage conditions, mixing types, and circumstance temperature could affect the viscosity of bone cement. Although this nomogram was useful and internally validated, external validation was also warranted. 
Table 5 Subgroup Analysis of Patients with Spine Metastases According to the Predicted Probability of Intra-Spinal Canal Cement Leaks

\begin{tabular}{|l|c|c|c|c|}
\hline Groups & Patients (n=163) & $\begin{array}{c}\text { Predicted } \\
\text { Probability }\end{array}$ & Actual Probability & $\mathbf{P}^{\mathbf{a}}$ \\
\hline Low risk group & 128 & $0.00-9.99 \%$ & $7.03 \%$ & $<0.01$ \\
Medium risk group & 26 & $10.00-24.99 \%$ & $11.54 \%$ & $44.44 \%$ \\
High risk group & 9 & $\geq 25.00 \%$ & \\
\hline
\end{tabular}

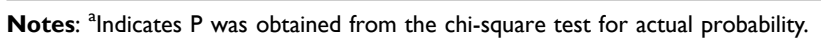

\section{Conclusions}

Intra-spinal canal cement leakage after PVP is not scarce among elderly patients. We proposed and internally validated a nomogram that is capable of calculating the risk of intra-spinal canal cement leakage among elderly patients with spine metastases. Careful surgical plan should be conducted among patients with a high risk of developing intra-spinal canal cement leakage.

\section{Abbreviations}

AUROC, area under the receiver operating characteristic curve; CI, confidence interval; CT, computed tomography; OR, odds ratio; PVP, percutaneous vertebroplasty.

\section{Data Sharing Statement}

The data are available upon reasonable request to the corresponding author.

\section{Ethics Approval and Consent to Participate}

The Ethics Committee Board of the Peking University First Hospital approved this study and waived the need for patient consent, because of the retrospective nature of the study and all data being anonymized.

\section{Funding}

There is no funding to report.

\section{Disclosure}

The authors declare that they have no conflict of interest.

\section{References}

1. Patchell RA, Tibbs PA, Regine WF, et al. Direct decompressive surgical resection in the treatment of spinal cord compression caused by metastatic cancer: a randomised trial. Lancet. 2005;366 (9486):643-648. doi:10.1016/S0140-6736(05)66954-1
2. Paulino Pereira NR, Ogink PT, Groot OQ, et al. Complications and reoperations after surgery for 647 patients with spine metastatic disease. Spine J. 2019;19(1):144-156. doi:10.1016/j. spinee.2018.05.037

3. Schuss P, Guresir A, Schneider M, Velten M, Vatter H, Guresir E. Factors influencing early postoperative complications following surgery for symptomatic spinal metastasis: a single-center series and multivariate analysis. Neurosurg Rev. 2020;43(1):211-216. doi:10.1007/s10143-018-1032-3

4. Tateiwa D, Oshima $K$, Nakai $T$, et al. Clinical outcomes and significant factors in the survival rate after decompression surgery for patients who were non-ambulatory due to spinal metastases. J Orthop Sci. 2019;24(2):347-352. doi:10.1016/j. jos.2018.10.003

5. Ozger O, Kaplan N. Clinical results of percutaneous vertebroplasty in thoracolumbar (T6-L5) vertebral compression fractures: retrospective study of 111 patients with 140 fractured segments. J Turk Spinal Surg. 2019;30(3):199-205.

6. Siu Y-C, Cheung M-H, Ma C-M. Intraspinal leakage of cement during vertebroplasty for an elderly woman with osteoporotic burst fracture: a case report and short review of prevention and management. J Orthop Traumatol Rehabil. 2015;19(2):100-106. doi:10.1016/j.jotr.2014.11.004

7. Zhang L, Wang Q, Wang L, Shen J, Zhang Q, Sun C. Bone cement distribution in the vertebral body affects chances of recompression after percutaneous vertebroplasty treatment in elderly patients with osteoporotic vertebral compression fractures. Clin Interv Aging. 2017;12:431-436. doi:10.2147/CIA.S113240

8. Ren H, Shen Y, Zhang YZ, et al. Correlative factor analysis on the complications resulting from cement leakage after percutaneous kyphoplasty in the treatment of osteoporotic vertebral compression fracture. J Spinal Disord Tech. 2010;23(7):e9-15. doi:10.1097/ BSD.0b013e3181c0cc94

9. Tome-Bermejo F, Pinera AR, Duran-Alvarez C, et al. Identification of risk factors for the occurrence of cement leakage during percutaneous vertebroplasty for painful osteoporotic or malignant vertebral fracture. Spine. 2014;39(11):E693-E700. doi:10.1097/ BRS.0000000000000294

10. Ding J, Zhang Q, Zhu J, et al. Risk factors for predicting cement leakage following percutaneous vertebroplasty for osteoporotic vertebral compression fractures. Eur Spine J. 2016;25(11):3411-3417. doi:10.1007/s00586-015-3923-0

11. Hulme PA, Krebs J, Ferguson SJ, Berlemann U. Vertebroplasty and kyphoplasty: a systematic review of 69 clinical studies. Spine. 2006;31(17):1983-2001. doi:10.1097/01.brs.0000229254.89952.6b

12. Zhang TY, Zhang PX, Xue F, Zhang DY, Jiang BG. Risk factors for cement leakage and nomogram for predicting the intradiscal cement leakage after the vertebra augmented surgery. BMC Musculoskelet Disord. 2020;21(1):792. doi:10.1186/s12891-020-03810-4 
13. Ahn JM, Oh JS. Gelfoam embolization technique to prevent bone cement leakage during percutaneous vertebroplasty: comparative study of Gelfoam only vs Gelfoam with venography. Korean J Neurotrauma. 2020;16(2):200-206. doi:10.13004/kjnt.2020.16.e42

14. Hsieh MK, Kao FC, Chiu PY, et al. Risk factors of neurological deficit and pulmonary cement embolism after percutaneous vertebroplasty. J Orthop Surg Res. 2019;14(1):406. doi:10.1186/ s13018-019-1459-4

15. Chen YJ, Tan TS, Chen WH, Chen CC, Lee TS. Intradural cement leakage: a devastatingly rare complication of vertebroplasty. Spine. 2006;31(12):E379-E382. doi:10.1097/01.brs.0000219495.57470.67

16. Tanigawa N, Kariya S, Komemushi A, et al. Cement leakage in percutaneous vertebroplasty for osteoporotic compression fractures with or without intravertebral clefts. AJR Am J Roentgenol. 2009;193 (5):W442-W445. doi:10.2214/AJR.09.2774

17. Kim YJ, Lee JW, Park KW, et al. Pulmonary cement embolism after percutaneous vertebroplasty in osteoporotic vertebral compression fractures: incidence, characteristics, and risk factors. Radiology. 2009;251(1):250-259. doi:10.1148/radiol.2511080854

18. Lei M, Li J, Liu Y, Jiang W, Liu S, Zhou S. Who are the best candidates for decompressive surgery and spine stabilization in patients with metastatic spinal cord compression? Spine. 2016;41 (18):1469-1476. doi:10.1097/BRS.0000000000001538

19. Gabriel C, Jonathan D, Charles M, et al. Cement leakage in percutaneous vertebroplasty for spinal metastases: a retrospective evaluation of incidence and risk factors. Spine. 2014;39(5):332-338. doi:10.1097/BRS.0000000000000134

20. Bilsky MH, Ilya L, Fourney DR, et al. Reliability analysis of the epidural spinal cord compression scale. J Neurosurg Spine. 2010;13 (3):324-328. doi:10.3171/2010.3.SPINE09459

21. Xiao S, Zhang L, Wu Q, et al. Development and validation of a risk nomogram model for predicting revascularization after percutaneous coronary intervention in patients with acute coronary syndrome. Clin Interv Aging. 2021;16:1541-1553. doi:10.2147/CIA.S325385
22. Yang Y, Zhu XF, Huang J, et al. Nomogram for prediction of fatal outcome in patients with severe COVID-19: a multicenter study. Mil Med Res. 2021;8(1):21.

23. Sun H, Yang Z, Xu Y, et al. Safety of percutaneous vertebroplasty for the treatment of metastatic spinal tumors in patients with posterior wall defects. Eur Spine J. 2015;24(8):1768-1777. doi:10.1007/ s00586-015-3810-8

24. Xie W, Jin D, Ma H, et al. Cement leakage in percutaneous vertebral augmentation for osteoporotic vertebral compression fractures: analysis of risk factors. Clin Spine Surg. 2016;29(4):E171-176. doi:10.1097/BSD.0000000000000229

25. Chen C, Fan P, Xie X, Wang Y. Risk factors for cement leakage and adjacent vertebral fractures in kyphoplasty for osteoporotic vertebral fractures. Clin Spine Surg. 2020;33(6):E251-E255. doi:10.1097/ BSD.0000000000000928

26. Liu T, Li Z, Su Q, Hai Y. Cement leakage in osteoporotic vertebral compression fractures with cortical defect using high-viscosity bone cement during unilateral percutaneous kyphoplasty surgery. Medicine. 2017;96(25):e7216. doi:10.1097/MD.0000000000007216

27. Zhu SY, Zhong ZM, Wu Q, Chen JT. Risk factors for bone cement leakage in percutaneous vertebroplasty: a retrospective study of four hundred and eighty five patients. Int Orthop. 2016;40(6):1205-1210. doi:10.1007/s00264-015-3102-2

28. Cui Y, Pan Y, Lei M, Mi C, Wang B, Shi X. The first algorithm calculating cement injection volumes in patients with spine metastases treated with percutaneous vertebroplasty. Ther Clin Risk Manag. 2020;16:417-428. doi:10.2147/TCRM.S253046

29. Janssen I, Ryang YM, Gempt J, et al. Risk of cement leakage and pulmonary embolism by bone cement-augmented pedicle screw fixation of the thoracolumbar spine. Spine J. 2017;17(6):837-844. doi:10.1016/j.spinee.2017.01.009

30. Baroud G, Crookshank M, Bohner M. High-viscosity cement significantly enhances uniformity of cement filling in vertebroplasty: an experimental model and study on cement leakage. Spine. 2006;31 (22):2562-2568. doi:10.1097/01.brs.0000240695.58651.62

\section{Publish your work in this journal}

Clinical Interventions in Aging is an international, peer-reviewed journal focusing on evidence-based reports on the value or lack thereof of treatments intended to prevent or delay the onset of maladaptive correlates of aging in human beings. This journal is indexed on PubMed Central, MedLine, CAS, Scopus and the Elsevier
Bibliographic databases. The manuscript management system is completely online and includes a very quick and fair peer-review system, which is all easy to use. Visit http://www.dovepress.com/ testimonials.php to read real quotes from published authors. 